\title{
Influence of megestrol acetate on nutrition, inflammation and quality of life in dialysis patients
}

\author{
Justyna E. Gołębiewska • Monika Lichodziejewska-Niemierko • \\ Ewa Aleksandrowicz-Wrona • Mikołaj Majkowicz • \\ Wiesława Łysiak-Szydłowska • Bolesław Rutkowski
}

Received: 19 March 2011/Accepted: 17 June 2011/Published online: 31 July 2011

(C) The Author(s) 2011. This article is published with open access at Springerlink.com

\begin{abstract}
Aim Malnutrition is a common clinical problem in dialysis patients. The objective of this study was to evaluate the efficacy and safety of megestrol acetate in malnourished dialysis patients. Thirty-two hypoalbuminemic dialysis patients took $160 \mathrm{mg}$ of megestrol acetate daily for up to 6 months.

Methods We measured height, dry weight, BMI, modified Subjective Global Assessment (SGA) score, and serum albumin, triglycerides, total cholesterol, hsCRP, IL- $1 \beta$ and IL-6 concentrations. We used validated questionnaires to evaluate selected dimensions of the quality of life.

Results Only 12 patients completed the study. All patients reported improved appetite, and there were
\end{abstract}

J. E. Gołębiewska $(\bowtie) \cdot$ M. Lichodziejewska-

Niemierko · B. Rutkowski

Department of Nephrology, Transplantology and Internal Medicine, Medical University of Gdańsk, ul. Dębinki 7, 80-952 Gdańsk, Poland

e-mail: jgolebiewska@gumed.edu.pl

M. Lichodziejewska-Niemierko

Palliative Medicine Department, Medical University

of Gdańsk, Gdańsk, Poland

E. Aleksandrowicz-Wrona - W. Łysiak-Szydłowska

Chair of Clinical Nutrition, Medical University

of Gdańsk, Gdańsk, Poland

M. Majkowicz

Quality of Life Research Department, Medical University of Gdańsk, Gdańsk, Poland concurrent statistically significant increases in weight, BMI, SGA and albumin concentration $(P<0.05)$. For the 12 patients who completed 6 months of treatment the increase in these parameters was from $63.26 \pm 13.04$ to $65.58 \pm 12.53 \mathrm{~kg}$, from $23.5 \pm 3.8$ to $24.66 \pm 4.23 \mathrm{~kg} / \mathrm{m}^{2}$, from $5.16 \pm 0.94$ to $6.16 \pm$ 0.72 points, and from $36.45 \pm 1.82$ to $40.33 \pm$ $2.71 \mathrm{~g} / \mathrm{l}$, respectively. However, there were no significant changes in the levels of inflammatory markers and in quality of life. Side effects included overhydration, excessive weight gain and hyperglycaemia.

Conclusion Megestrol acetate may be effective in reversing poor appetite in carefully selected maintenance dialysis patients, but it might not reduce inflammation or improve the quality of life. Because of the potential side effects, close monitoring is essential.

Keywords Malnutrition - Megestrol acetate · Proinflammatory cytokines · Dialysis · Quality of life

\section{Introduction}

The number of end-stage renal disease (ESRD) patients is constantly increasing, including those requiring chronic renal replacement therapy. Despite the huge headway that has been made in dialysis techniques and equipment, the morbidity burden faced by patients maintained on dialysis still remains 
unacceptably high. Among the most common problems experienced by these patients are anorexia and protein-energy malnutrition or wasting [1]. Their prevalence varies depending on the study, within the range of $10-75 \%$ [2,3]. Malnutrition is a problem not only because of its negative impact on quality of life $[4,5]$, comorbidities and hospitalizations, but also, more importantly, its influence on cardiovascular and all-cause mortality [5]. Successful management of malnutrition could therefore ameliorate the cardiovascular epidemic and poor outcomes in dialysis patients $[2,6]$.

The aetiology of malnutrition in ESRD is complex and includes a range of underlying factors. Hence, its treatment is difficult. Protein-energy malnutrition often overlaps with inflammation and atherosclerosis leading to MIA (malnutrition-inflammation-atherosclerosis) syndrome [2, 7]. Proinflammatory cytokines, such as IL-1, IL-6, and TNF- $\alpha$, play a key role in these events. This is why treatment aimed at eliminating or reducing inflammation becomes a necessity when conventional nutritional and metabolic interventions do not provide satisfactory results. Several new strategies, including the use of megestrol acetate, are under investigation.

Megestrol acetate is a synthetic progestagen that was shown to increase appetite and body weight in patients with cancer and AIDS and improves quality of life in patients with cancer [8]. It is believed to work by down-regulating proinflammatory cytokines as IL-1, IL-6, and TNF- $\alpha$ [9, 10]. It could therefore improve not only nutritional status, but also reverse cytokine-dependent inflammatory processes. In recent years, quality of life, i.e. the patient's functional status and subjective state of well-being, has been recognized as one of the important measures of the outcome of medical treatment.

To date, there have been relatively few evaluations of megestrol acetate therapy in dialysis patients [11-17]. These limited, but promising studies, with variable results, the successful use of megestrol acetate in cancer and AIDS patients, and the lack of data regarding the influence of megestrol acetate on inflammation in ESRD patients, support the rationale for a new study in the latter. The goal of this study was to examine the influence of $160 \mathrm{mg}$ daily intake of megestrol acetate (for 6 months) on nutrition, inflammation and quality of life in dialysis patients.
The dose of $160 \mathrm{mg}$ was chosen, as it was reported to be safe, yet effective.

\section{Patients and methods}

Subject recruitment and study design

We used a multicenter, prospective design. The study protocol was approved by the Bioethics Committee of the Medical University of Gdańsk. All procedures were performed in accordance with the Declaration of Helsinki.

We screened MHD (maintenance haemodialysis) and CPD (chronic peritoneal dialysis) patients from six haemodialysis facilities (381 patients) and two peritoneal dialysis centres (89 patients). Inclusion criteria were the following: maintenance dialysis treatment for at least 3 months and serum albumin concentration $\leq 3.8 \mathrm{~g} / \mathrm{dl}$ (Bromcresol Green). Exclusion criteria were concurrent use of glucocorticoids and inadequate dialysis as defined by a $\mathrm{Kt} / \mathrm{V}$ of $<1.2$ for MHD and $<2.0$ for CPD patients. HIV/ AIDS or malignancy were not exclusion criteria. Before enrolment, patients received detailed written and verbal information about the aims and protocol of the study. Patients gave written informed consent.

We measured anthropometric parameters and carried out the biochemical analyses at baseline and every month thereafter. We assessed SGA and asked patients to complete the quality of life questionnaires at baseline and every 3 months thereafter.

Patients were provided with megestrol acetate solution (Megalia, Vipharm) after the baseline measurements and instructed to take $4 \mathrm{ml}(160 \mathrm{mg})$ daily. On haemodialysis days, MHD patients brought in their bottles for weighing and were witnessed taking the drug. On the remaining days, they took the drug at home. CPD patients brought in their bottles for weighing and were asked to return the empty bottles on every visit.

Anthropometric indices

These included height, dry weight, and body mass index (BMI). The BMI was calculated using the following equation: dry weight $(\mathrm{kg})$ : height squared $\left(\mathrm{m}^{2}\right)$. 
Biochemical analysis (nutritional and inflammatory indices)

Blood sample was drawn from a peripheral vein (in the CPD patients) or from the artero-venous fistula or catheter before dialysis (in the MHD patients). Serum was separated $<30 \mathrm{~min}$ after drawing and stored at $-70^{\circ} \mathrm{C}$ until analysis. Standard laboratory techniques were used for the determination of serum albumin, triglycerides and total cholesterol concentrations (Abbott Clinical Chemistry). The hsCRP and cytokines (IL-1 $\beta$ and IL-6) concentrations were measured by high-sensitivity sandwich enzyme-linked immunosorbent assay (ELISA) kits (DRG and BenderMed Systems, respectively).

\section{Subjective global assessment}

We used a subjective global assessment using a quantitative scoring system consisting of seven variables: weight change, dietary intake, gastrointestinal symptoms, functional capacity, comorbidity, subcutaneous fat and signs of muscle wasting. Each item was scored from 1 to 7 points, where 1 represented severely malnourished and 7 well nourished.

\section{Quality of life analysis}

Selected dimensions of the Quality of Life (QoL) were evaluated using validated questionnaires. The level of negative emotions (depression, anxiety and aggression) was measured on the modified Hospital Anxiety and Depression Scale. The intensity of positive emotions (purpose and meaning of life perception) was evaluated with the Purpose in Life Test (PIL). Cantril's Ladder was used to measure life satisfaction, and fatigue was evaluated using the Brief Fatigue Inventory (BFI). Patients completed the questionnaires during or before dialysis sessions or during scheduled outpatient visits at the PD centre. Assistance with filling in the forms was provided if necessary.

\section{Statistics}

All analyses were performed using Statistica 8.0 (StatSoft) and StatsDirect 7.6.5 software. Data are presented as mean $\pm \mathrm{SD}$, unless otherwise indicated. $P<0.05$ was considered to be significant. To assess the normalcy of the distributions, we used the Kolmogorow-Smirnow and Lilliefors tests. To assess the equality of variance, we used Fisher's F test and Levene's test. The Spearman's or, where appropriate, Pearson's rank order correlation coefficient were used to explore the relationship between indices at baseline. To assess the significance of changes of the repeated measures, either parametric or Friedman's ANOVA were used. Post hoc analyses were the Least Significant Differences test and Conover test.

\section{Results}

Baseline characteristics of patients

Forty-nine MHD and 19 CPD patients of the total of 381 MHD and 89 CPD patients were eligible for the study. Thirty-four MHD and 11 CPD patients agreed to participate and were enrolled. Thirteen patients had dropped out before the study started or within the first 4 weeks of the study; nine of these withdrew their consent without giving an explanation, two underwent renal transplantation, one died on the fourth week of the study of causes unrelated to the study (cholangitis leading to sepsis) and one was excluded from the study because of noncompliance. Data from these individuals were not included in the analysis.

The analysis involves 32 patients (24 MHD and 8 CPD; 18 men and 14 women) who had completed at least 1 month of the study. The mean age of patients was $69.97 \pm 10.8$ years (range 38-85), and the mean dialysis vintage was $33.55 \pm 35.7$ months (range $=$ 3-133). The causes of ESRD were the following: diabetic nephropathy ( 9 patients), glomerulonephritis (7), hypertensive nephropathy (3), interstitial nephritis (3), polycystic kidney disease (2) and others (8). None of patients had HIV infection and/or active neoplastic disease. However, there were three patients with a history of cancer. One patient underwent bilateral nephrectomy due to renal cancer 4 years prior to inclusion in the study, one underwent single kidney resection due to renal cancer 18 months before the study started and one was submitted to laryngectomy because of larynx cancer 8 years earlier.

Thirty-four per cent of all included patients with albuminaemia $<38 \mathrm{~g} / \mathrm{l}$ had albuminaemia $<35 \mathrm{~g} / \mathrm{l}$, $31 \%$ suffered from inflammation (hsCRP $\geq 10 \mathrm{mg} / \mathrm{l}$ ), $56 \%$ from CVD and two patients had a combination of 
these three components and were therefore diagnosed with MIA syndrome.

All 32 patients included in the analysis suffered from mild-to-moderate malnutrition according to SGA evaluation at baseline, with median of five points and six points as maximum and three as minimum score.

Before megestrol acetate treatment was started, we found no statistically significant correlations between laboratory indices of nutrition and inflammation. However, there were significant correlations between various measures used for the assessment of nutritional status (SGA, BMI, albuminaemia) and between hsCRP and IL-6.

Participation period and premature termination of participation

The mean participation period was $3.5 \pm 2.1$ (median 2.5) months. Thirty-two patients completed 1 month of the study, 26 patients completed 2 months of the study, 18 completed 3 months, 14 completed 4 months and 12 each completed 5 and 6 months. Reasons for premature termination were the following: death unrelated to the treatment (7), renal transplantation (1), withdrawn consent (1) and side effects (11) (see Fig. 1 for patient flowchart).

\section{Nutritional indices}

All patients reported improved appetite, which was accompanied by a substantial increase in the daily energy intake. In the group of 32 patients who completed the first 4 weeks of the study, we noticed no significant increase in body weight or BMI. In the 26 patients who completed at least 2 months of megestrol acetate therapy, there was a significant increase in BMI from $23.06 \pm 3.26 \mathrm{~kg} / \mathrm{m}^{2}$ at baseline to $23.68 \pm 3.63 \mathrm{~kg} / \mathrm{m}^{2}(P=0.03)$, but there was no significant change in body weight. From the third month of the trial onwards, changes in both of these measures became statistically significant $(P<0.05)$.

An increase in serum albumin concentration became statistically significant 1 month into the intervention period (from $35.15 \pm 3.28$ to $37.28 \pm 3.77 \mathrm{~g} /$ 1; $P<0.05)$. The increase persisted throughout the 6 months of the study. A trend towards a decrease in the concentration of total cholesterol $(P=0.052)$ was seen during the first 2 months of treatment, but not afterwards. There was also a statistically significant change in SGA, both in the 18 patients who completed 3 months $(P=0.003)$ and in the 12 who completed the whole treatment $(P<0.002)$. There was no change in the concentration of triglycerides. The results after 2 and 6 months are presented in Tables 1 and 2, respectively.

In the 26 patients who completed 2 months of treatment, post hoc analysis revealed that the minimum treatment period required to reach a statistically significant increase in weight gain was 2 months. A significant change in BMI was seen after 2 months of treatment with megestrol acetate and between the first and second months of the treatment. The albumin concentration increased significantly over the first 4 weeks and the first 2 months of the intervention period. However, the changes between the fourth and eighth week of the study were insignificant. In the 12 patients who took megestrol acetate for 6 months, the increase in body weight and BMI reached significance after at least 4 and 3 months of treatment, respectively. However, the albumin concentration had increased significantly by 1 month.

There was no relation between the dialysis modality (peritoneal vs. haemodialysis) and the change in nutritional status during treatment. Weight gain was significantly worse in patients with diabetes, while having diabetes did not influence the change in the albumin concentration.

Inflammatory indices

There were no statistically significant changes in the concentrations of hsCRP, IL- $1 \beta$ or IL- 6 throughout the whole study period (Tables 1 and 2).

\section{Quality of life}

Quality of life was estimated in the 18 patients who completed 3 months and the 12 who completed 6 months of treatment. There were no significant changes in valuation of life satisfaction, fatigue or intensity of positive emotions, purpose and meaning of life perception, and negative emotions.

Side effects of therapy

Side effects were common. Overhydration of varying severity or excessive fluid gain $(>4 \mathrm{~kg}$ interdialytic 


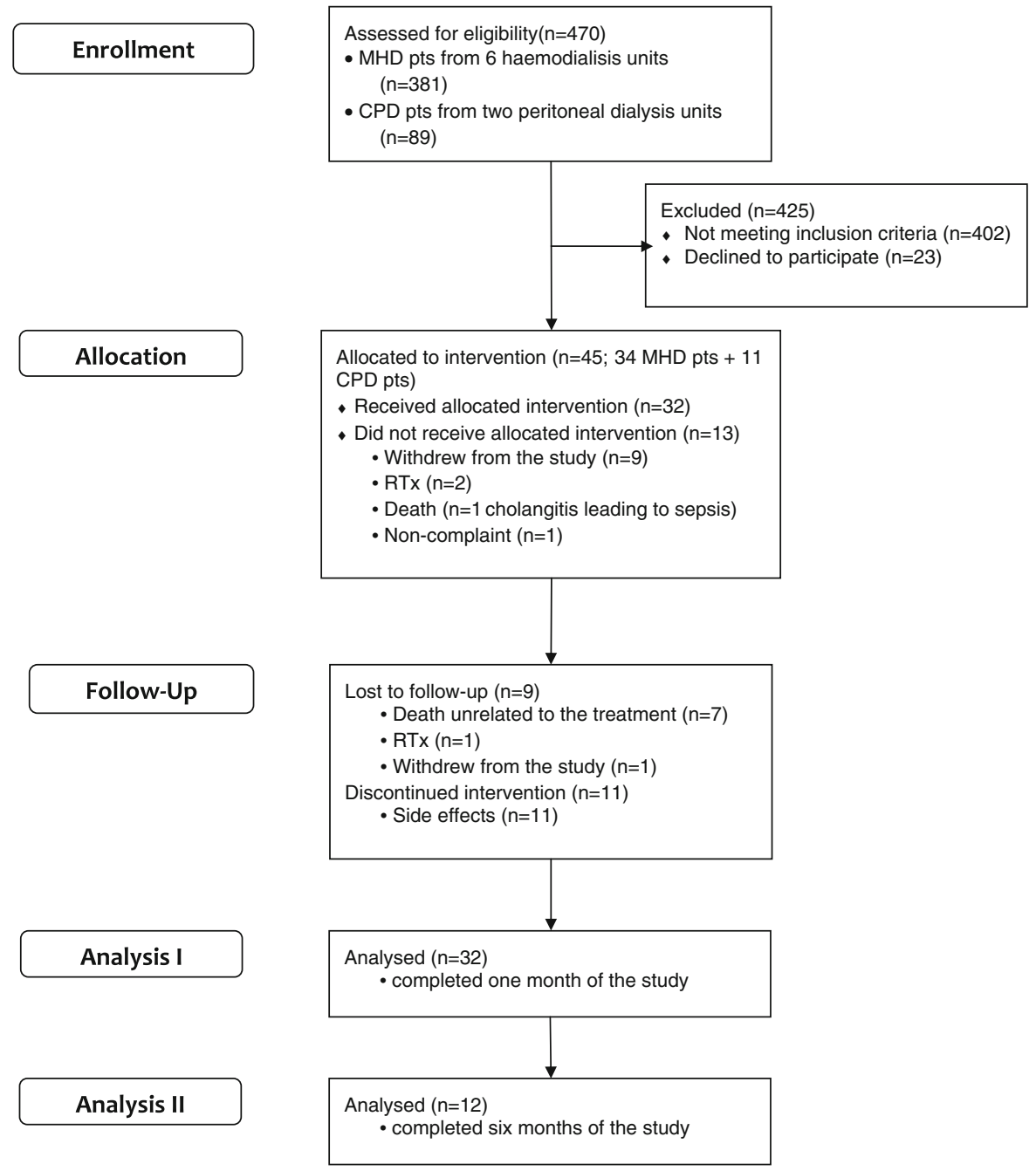

Fig. 1 Patient flowchart

Table 1 Nutritional and inflammatory indices before and after 1 and 2 months of megestrol acetate administration $(n=26)$

\begin{tabular}{lcccc}
\hline Variable & Baseline $($ mean $\pm \mathrm{SD})$ & 1 month $($ mean $\pm \mathrm{SD})$ & 2 month $(\mathrm{mean} \pm \mathrm{SD})$ & $P$ value \\
\hline Weight $(\mathrm{kg})$ & $63.58 \pm 12.38$ & $64.22 \pm 12.1$ & $64.78 \pm 11.97$ & $\mathrm{NS}(0.16)$ \\
BMI $\left(\mathrm{kg} / \mathrm{m}^{2}\right)$ & $23.06 \pm 3.26$ & $23.34 \pm 3.3$ & $23.68 \pm 3.63$ & 0.03 \\
Albumin $(\mathrm{g} / \mathrm{dl})$ & $35.46 \pm 2.88$ & $37.42 \pm 3.44$ & $36.8 \pm 4.44$ & 0.01 \\
Total cholesterol $(\mathrm{mg} / \mathrm{dl})$ & $173.96 \pm 57.66$ & $168.42 \pm 51.85$ & $165.27 \pm 57.46$ & $\mathrm{NS}(0.052)$ \\
Triglicerydes $(\mathrm{mg} / \mathrm{dl})$ & $143.42 \pm 73.6$ & $136.38 \pm 59.13$ & $131.11 \pm 57.42$ & $\mathrm{NS}$ \\
HsCRP $(\mathrm{mg} / \mathrm{l})$ & $6.98 \pm 5.38$ & $6.99 \pm 5.74$ & $10.2 \pm 7.22$ & $\mathrm{NS}$ \\
IL-1 $\beta(\mathrm{pg} / \mathrm{ml})$ & $2.42 \pm 4.65$ & $1.91 \pm 3.17$ & $1.52 \pm 1.63$ & $\mathrm{NS}$ \\
IL-6 $(\mathrm{pg} / \mathrm{ml})$ & $24.2 \pm 18.82$ & $35.77 \pm 39.05$ & $33.03 \pm 46.89$ & $\mathrm{NS}$ \\
\hline
\end{tabular}

weight gain or/and overhydration causing pulmonary congestion and dyspnoea) between haemodialysis sessions was seen in nine patients, and a dose reduction of megestrol acetate was required in all. Two patients withdrew from the study after 2 months and one after 4 months. One of the signs of overhydration was a raise 


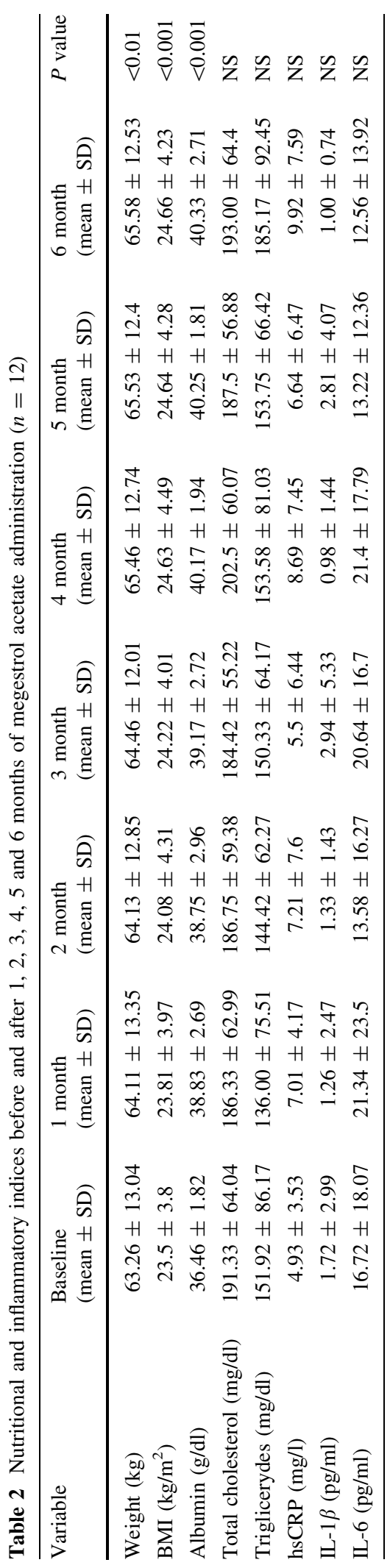

in blood pressure. However, none of the patients without symptomatic overhydration developed hypertension or required modification of antihypertensive treatment. Excessive weight gain ( $>5 \%$ of initial body weight within 4 weeks) developed in six subjects, and in four of these a dose reduction was necessary. Two patients withdrew from the study after 2 and 3 months, respectively. One of the patients with diabetes required a dose reduction because of hyperglycaemia. This was difficult to control by increasing the dose of insulin. Diarrhoea in three patients and nausea and vomiting in three subjects led to discontinuation of the megestrol acetate. Another two patients withdrew prematurely because of thrombophlebitis. Some patients experienced more than one side effect; most commonly excessive weight gain was accompanied by overhydration.

\section{Discussion}

Dialysis is an effective treatment that prolongs the lives of ESRD patients but at the same time brings them an enormous burden. One of the most common clinical problems faced by dialysis patients are anorexia and malnutrition. Their negative impact on the quality of life, number of comorbidities and hospitalizations, and, most importantly, its deleterious influence on cardiovascular and all-cause mortality are widely recognized. Dialysis patients themselves perceive anorexia as one of the most unpleasant of all symptoms $[18,19]$. So far, the search for an effective and safe therapeutic agent that could be used to correct anorexia and/or malnutrition has not been successful. Hence, we decided to evaluate the influence and safety of megestrol acetate on nutrition, inflammation and quality of life in this population.

Even though malnutrition is a common clinical problem and a strong predictor of cardiovascular and all-cause mortality in dialysis patients, there is still no single test that reliably estimates the severity of protein-energy malnutrition. An accurate assessment of nutritional status in dialysis patients is therefore difficult. We decided to apply a commonly used, simple and inexpensive marker of malnutrition, serum albumin concentration $\leq 38 \mathrm{~g} / \mathrm{l}$. At the same time, we were aware of the fact that inflammatory states, plasma volume and distribution between extra- and intravascular compartments limit the specificity of serum albumin as a nutritional marker. 
According to our definition, only $14.5 \%$ of almost 500 patients screened were hypoalbuminemic. One-third of these suffered from microinflammation, and about 50\% had cardiovascular diseases. Only two patients had all three components of MIA syndrome concurrently. This suggests that the actual prevalence of malnutrition and MIA syndrome among dialysis patients may have been overestimated or that serum albumin concentration is not a perfect tool for assessing malnutrition. The former is consistent with a recent study [20]. In this study, only $10 \%$ of patients were malnourished 3 months after the start of chronic haemodialysis treatment. In our study, one of the inclusion criteria was maintenance dialysis treatment for at least 3 months. In addition, the DOPPS study found significant differences between countries in the proportion of moderately and severely malnourished dialysis patients on the basis of various nutritional markers including serum albumin [21]. In a more recent study, hypoalbumineamia (defined as $<3.5 \mathrm{~g} / \mathrm{dl}$ ) was found in only $8 \%$ of a group of 149 MHD patients from a single dialysis centre [22]. On the other hand, despite having acceptable level of albumin, dialysis patients have abnormalities in their amino acid profiles indicating malnutrition [23].

We found that in hypoalbuminemic MHD and CPD patients $160 \mathrm{mg}$ of an oral suspension of megestrol acetate taken daily for up to 6 months improved several markers of nutritional state but had no influence on inflammation or quality of life. The most impressive result was the significant increase in albumin concentration beginning from the first month and continuing throughout the whole trial period.

Significant weight gains and increases in BMI were noted in the following months. Weight gain was significantly lower in diabetics, while the rate of increase in serum albumin concentration remained unaffected. Unlike the presence of diabetes, the mode of dialysis did not influence the changes in nutritional status. Side effects of therapy were prevalent and significant.

To date, only seven studies evaluating the use of megestrol acetate in dialysis patients have been published. Burrowes et al. [11] published a case study of a haemodialysis patient taking megestrol acetate $320 \mathrm{mg} /$ day for 24 weeks. The patient reported an improvement in appetite, and his fat mass increased by $163 \%$, whereas his lean mass decreased by $10.6 \%$. Lien et al. [12] placed 12 chronic peritoneal dialysis (CPD) and four maintenance haemodialysis (MHD) hypoalbuminemic patients $(<3.5 \mathrm{~g} / \mathrm{dl}$ for two consecutive months $)$ on megestrol acetate $20 \mathrm{mg}$ twice daily. Serum albumin increased in $75 \%$ of patients. One patient stopped taking the drug because of vaginal bleeding from leiomyoma.

Boccanfuso et al. [13] evaluated the effect of megestrol acetate suspension $400 \mathrm{mg}$ orally twice daily for up to 6 months in 17 MHD patients with serum albumin $<3.5 \mathrm{~g} / \mathrm{dl}$ for two consecutive months. At the end of the third month, only $53 \%$ of those patients were still in the study, whereas only three of them were able to take the studied medicine for 5 to 6 months. Each of these three patients had a positive change in SGA, appetite and caloric intake and an increase in dry weight. Side effects leading to dropouts were common and included diarrhoea, confusion, headache and hyperglycaemia.

Costero et al. [14] treated 32 CPD patients with $160 \mathrm{mg}$ of megestrol acetate daily for 1-23 months and observed an increase in appetite in most patients, together with a significant weight gain and no significant change in serum albumin. No side effects were reported. Rammohan et al. [15] assessed the efficacy of $400 \mathrm{mg}$ of megestrol acetate taken daily for 16 weeks in nine MHD and one CPD patients. Appetite, daily energy intake, body weight and serum albumin increased significantly. There was also a fall in serum CRP concentration of borderline significance. Quality of life was also reported to be improved. No major side effects were observed.

Recently, Monfared et al. [16] published the results of a randomized controlled trial in a group of hypoalbuminemic haemodialysis patients. Eleven patients assigned to experimental group were treated with $40 \mathrm{mg}$ of megestrol acetate twice daily for 2 months, while 11 patients in the control group continued with their previous treatment. There was a statistically significant increase in serum albumin concentration in the treatment group with concurrent changes in PCR level, while there were no significant changes in the control group. However, these authors reported no changes in the total pre- and post-dialysis body weight of patients. Recently, Yeh et al. [17] demonstrated in their double-blind, placebocontrolled study with $800 \mathrm{mg}$ megestrol acetate daily combined with progressive resistance physical therapy, that megestrol acetate use in a group of nine 
elderly, cachectic haemodialysis patients lead to improvement in weight, with favourable increase in fat mass over lean body mass. Simultaneously, no statistically significant changes in inflammation markers apart from a decrease in hsCRP level and an insignificant improvement in the sense of wellbeing were seen.

The exact mechanism by which megestrol acetate exerts its orexigenic effect is unknown. It has been suggested that it could be due to down-regulation of the synthesis and secretion of proinflammatory cytokines. If so, the possession of both appetitestimulating and anti-inflammatory properties would make megestrol acetate an optimal tool for the treatment of malnutrition and MIA syndrome. However, we saw no significant changes in hsCRP, IL-1 $\beta$ or IL-6 concentrations after megestrol acetate treatment, while Rammohan et al. [15] described a decrease in the hsCRP concentration that was of borderline significance. Similar results regarding IL-6 were reported by Jatoi et al. [24] in a group of 85 patients with cancer-associated anorexia or weight loss. The authors suggested that other mechanisms were responsible for the favourable effect of megestrol acetate. Megestrol acetate possesses both glucocorticoid and progestogenic activities [25]. The results from animal studies show that megestrol acetate stimulation of appetite may involve neuropeptide Y (NPY) [26] and/or calcium channels in ventromedial hypothalamus, a well-known satiety centre [27]. The NPY-induced feeding is known to be dependent on circulating glucocorticoid levels. On the other hand, feeding behaviour in women is influenced by the menstrual cycle, with the highest food intake during the post-ovulatory luteal phase, when progesterone levels are highest [28].

Wallace et al. [29] also reported similar findings: the concentrations of IL-6 and CRP remained unchanged after 6-12 weeks exposure to megestrol acetate in malnourished gastrointestinal cancer patients. Furthermore, the levels of inflammatory biomarkers such as CRP, IL-6 and fetuin A show considerable intrapatient fluctuations (31-46\%), even in apparently clinically healthy dialysis patients. These markers might be of limited value as surrogate endpoints in clinical trials [30].

There is also a within-subject variation of albumin concentration. Even though both albumin and CRP are acute-phase reactants, the variations of CRP are two orders greater than that of albumin. This phenomenon could be attributed to longer half-life, higher molecular mass and much smaller biological range of albumin synthesis rates, fractional catabolic rates and serum values [31]. Consequently, withinsubject fluctuations cannot be ruled out as the cause of interference with the general trend in a small study population and therefore makes it impossible to draw statistically significant conclusions.

There is another possible explanation for the lack of decrease in proinflammatory cytokines concentration in spite of the anti-inflammatory properties of megestrol acetate. Adipose tissue is an active endocrine organ secreting proinflammatory adipokines such as IL-6 [32]. In 43 peritoneal dialysis patients, BMI correlated with the levels of leptin, CRP, IL-6 and $\mathrm{TNF} \alpha$ [33]. In a group of 197 ESRD patients, individuals with higher total body and truncal fat mass had higher CRP, fibrinogen and IL-6 levels [34]. In the same study, there was a negative correlation between IL-6 and handgrip strength. Similar observations were made in CKD stage 3 and stage 4 patients [35]. Honda et al. [36] found that, in a group of 328 ESRD patients, CRP, IL-6 and $\mathrm{TNF} \alpha$ were highest in wasted patients with BMI $>25 \mathrm{~kg} / \mathrm{m}^{2}$. These patients tended to be older, with a higher prevalence of comorbidities, especially CVD and diabetes mellitus. These characteristics also applied to our study population.

To date, analyses of body composition in dialysis patients taking megestrol acetate have shown a statistically significant increase in fat mass with a concurrent decrease in lean body mass $[11,15]$. Also, in AIDS and cancer patients treated with megestrol acetate, the increase in fat mass outweighs by far the minimal improvements in protein balance. This could explain the statistically significant increase in body mass without any simultaneous increase in albuminaemia described previously in dialysis patients taking megestrol acetate. Therefore, the amount of proinflammatory cytokines secreted will increase as the fat mass increases, despite down-regulation by an unchanged dose of megestrol acetate.

This hypothesis seems to contradict the reverse epidemiology phenomenon and the protective effect of high BMI in dialysis patients. However, Honda et al. [36] found that high BMI was associated with a survival advantage, provided that it was not accompanied by protein-energy wasting. Similarly, 
Beddhu et al. [37] reported that the protective effect conferred by high BMI was limited to those patients with normal or high muscle mass in a group of more than 70,000 haemodialysis patients. Patients with BMI $\geq 25 \mathrm{~kg} / \mathrm{m}^{2}$ and low muscle mass had a higher risk of all-cause and cardiovascular death compared to patients with BMIs ranging from 18.5 to $24.9 \mathrm{~kg} / \mathrm{m}^{2}$ and normal or high muscle mass.

A significant increase in albumin concentration can occur together with muscle mass depletion. Lean body mass, as determined by creatinine kinetics [38], anthropometry and bioelectrical impedance, correlated poorly or not at all with serum albumin level [39]. Megestrol acetate has glucocorticoid-like activity $[25,40]$, and glucocorticoids are known to upregulate albumin gene expression in vitro [41] and to increase albumin synthesis in vivo [42], while stimulating muscle protein breakdown. Albumin is a negative acute-phase protein, but its concentration and synthesis rate are also determined by numerous factors other than inflammation [43]. For that reason, megestrol acetate improved nutritional status, as measured by total body mass and serum albumin, but did not reduce inflammation.

As mentioned earlier, megestrol acetate favours an increase in fat mass with a concurrent depletion of lean body mass. Muscle mass loss has been shown to increase the mortality risk in predialysis CKD stage 5 patients [44], haemodialysis patients [44-47], haemodiafiltration patients [48] and peritoneal dialysis patients [39, 49]. It would therefore be reasonable to combine megestrol acetate treatment with another intervention that would result in more favourable body composition changes.

Because megestrol acetate induces hypogonadism, there have been unsuccessful attempts to supplement megestrol acetate treatment with testosterone in men with HIV-associated weight loss, in order to promote lean body mass [50]. Another option would be to add resistance or aerobic training. The rationale for introducing an exercise programme is that positive energy balance during inactivity has been shown to be associated with greater muscle atrophy and activation of systemic inflammation [51].

Megestrol acetate has been reported to improve quality of life in cancer patients. Malnourished dialysis patients often show substantial reduction in quality of life, thus valuation of how different dimensions of QoL are affected by megestrol acetate was an integral part of the study. As hypoalbuminaemia has been associated with poorer quality of life [4, 5, 52-54], an intervention leading to an increase in serum albumin concentration would be expected to improve health-related QoL. Nevertheless, we saw no significant changes in QoL. Although this may contradict the assumed association between albumin level and QoL, it should be kept in mind that megestrol acetate increases fat mass with a relatively small improvement in protein balance. Our findings are consistent with the results of Goller et al. [55], who reported that overweight CPD patients had lower SF 36 scores. Similarly, Kalantar-Zadeh et al. [56] examined the relationship between SF 36 and body fat percentage in 535 MHD patients and found that overweight individuals perceive a worse QoL.

An alternative explanation is provided by SimicOgrizovic et al. [57], who investigated the association between depression and various clinical indices in $128 \mathrm{MHD}$ and CPD patients. Depression was closely associated with malnutrition, inflammation and cardiovascular mortality, but the most powerful variable associated with depression was IL-6. The lack of a decrease in proinflammatory cytokine concentrations possibly resulted in unchanged levels of negative emotions.

Another issue is the high prevalence of significant side effects, making it necessary to reduce the dose or to end the planned treatment prematurely. This was especially surprising, as the $160 \mathrm{mg}$ dose we decided to use has been described to be safe and associated with no adverse events [14]. It is difficult to explain the lack of side effects when using higher doses of megestrol acetate [15]. The most common and the most alarming was overhydration of varying severity or excessive fluid gain between haemodialysis sessions. In a study by Kalantar-Zadeh et al. [58], greater fluid retention between two consecutive haemodialysis sessions was associated with a higher risk of all-cause and cardiovascular death, even though higher weight gain was associated with better nutritional status. Furthermore, the fluid retentionsurvival association was stronger in individuals with higher albuminaemia.

The main drawback of our study was the lack of a control group. This was due to a very limited number of patients who agreed to participate (32 patients out of 490 patients screened). Technical limitations also meant that we could not analyse body composition 
changes. The use of visual analogue scale for appetite assessment and measurement of actual food intake would have allowed a more accurate estimation of the orexigenic effect. However, patients would have had to fill in more questionnaires and this could have adversely affected the QoL assessment.

In conclusion, megestrol acetate may be effective in reversing poor appetite in carefully selected maintenance dialysis patients. It improves some measures of nutritional status, but does not reduce inflammation and does not improve quality of life. There is virtually no evidence that megestrol acetateinduced weight gain or that stimulating appetite and improving selected nutritional indices in patients with chronic disease wasting confers any survival benefit. No data exist to indicate that the composition of weight gain affects the outcome. Tailoring the dosage according to individual response could possibly solve some megestrol-related problems. However, due to serious and prevalent side effects and its unproven QoL benefits, its use must be considered and managed carefully and monitored closely [59], especially in dialysis patients. Further studies are needed to investigate whether the benefits of megestrol acetate outweigh its side effects.

Acknowledgments Ministry of Science and Higher Education grant no. N 402007 32/0121.

\section{Conflict of interest None.}

Open Access This article is distributed under the terms of the Creative Commons Attribution Noncommercial License which permits any noncommercial use, distribution, and reproduction in any medium, provided the original author(s) and source are credited.

\section{References}

1. Murtagh FE, Addington-Hall J, Higginson IJ (2007) The prevalence of symptoms in end-stage renal disease: a systematic review. Adv Chronic Kidney Dis 14:82-99

2. Kalantar-Zadeh K, Ikizler A, Block G et al (2003) Malnutrition-inflammation complex syndrome in dialysis patients: causes and consequences. Am J Kidney Dis 42:864-881

3. Fouque D, Kalantar-Zadeh K, Kopple J et al (2008) A proposed nomenclature and diagnostic criteria for proteinenergy wasting in acute and chronic kidney disease. Kidney Int 73:391-398
4. Dwyer JT, Larive B, Leung J et al (2002) Nutritional status affects quality of life in hemodialysis (HEMO) study patients at baseline. J Ren Nutr 12:213-223

5. Kalantar-Zadeh K, Kopple J, Block G et al (2001) Association among SF36 quality of life measures and nutrition, hospitalization, and mortality in hemodialysis. J Am Soc Nephrol 12:2797-2806

6. Kalantar-Zadeh K, Kilpatrick RD, Kuwae N et al (2005) Revisiting mortality predictability of serum albumin in the dialysis population: time dependency, longitudinal changes and population-attributable fraction. Nephrol Dial Transplant 20:1880-1888

7. Rashid Qureshi A, Alvestrand A, Divino-Filho JC et al (2002) Inflammation, malnutrition, and cardiac disease as predictors of mortality in hemodialysis patients. J Am Soc Nephrol 13:S28-S36

8. Lopez AP, Figuls MR, Cuchi GU et al (2004) Systematic review of megestrol acetate in the treatment of anorexiacachexia syndrome. J Pain Symptom Manage 27:360-369

9. Mantovani G, Maccio A, Lai P et al (1998) Cytokine activity in cancer-related anorexia/cachexia: role of megestrol acetate and medroxyprogesterone acetate. Semin Oncol 25:45-52

10. Mantovani G, Maccio A, Lai P et al (1998) Cytokine involvement in cancer anorexia/cachexia: role of megestrol acetate and medroxyprogesterone acetate on cytokine downregulation and improvement of clinical symptoms. Crit Rev Oncol 9:99-106

11. Burrowes JD, Bluestone PA, Wang J, Pierson RN Jr (1999) The effects of moderate doses of megestrol acetate on nutritional status and body composition in a hemodialysis patient. J Ren Nutr 9:89-94

12. Lien YH, Ruffenach SJ (1996) Low dose megestrol increases serum albumin in malnourished dialysis patients. Int J Artif Organs 19:147-150

13. Boccanfuso JA, Hutton M, McAllister B (2000) The effects of megestrol acetate on nutritional parameters in a dialysis population. J Ren Nutr 10:36-43

14. Costero O, Bajo MA, del Peso G et al (2004) Treatment of anorexia and malnutrition in peritoneal dialysis patients with megestrol acetate. Adv Perit Dial 20:209-212

15. Rammohan M, Kalantar-Zadeh K, Liang A, Ghossein C (2005) Megestrol acetate in a moderate dose for the treatment of malnutrition-inflammation complex in maintenance dialysis patients. J Ren Nutr 15:345-355

16. Monfared A, Heidarzadeh A, Ghaffari M, Akbarpour M (2009) Effect of megestrol acetate on serum albumin level in malnourished dialysis patients. J Ren Nutr 19:167-171

17. Yeh SS, Marandi M, Thode HC et al (2010) Report of a pilot, double-blind, placebo-controlled study of megestrol acetate in elderly dialysis patients with cachexia. J Ren Nutr 20:52-62

18. Majkowicz M, Afeltowicz Z, Dębska-Ślizień A et al (1999) Jakość życia chorych hemodializowanych, dializowanych otrzewnowo oraz pacjentów onkologicznych. Psychonkologia 4:53-63 (in Polish)

19. Majkowicz M, Afeltowicz Z, Lichodziejewska-Niemierko $M$ et al (2000) Comparison of the quality of life in hemodialysed (HD) and peritoneallydialysed (CAPD) patients using the EORTC QLQ-C30 questionnaire. Int $\mathbf{J}$ Artif Organs 23:423-428 
20. De Mutsert R, Grootendorst DC, Axelsson J, Boeschoten EW, Krediet RT, Dekker FW, The NECOSAD Study Group (2008) Excess mortality due to interaction between protein-energy wasting, inflammation and cardiovascular disease in chronic dialysis patients. Nephrol Dial Transplant 23:2957-2964

21. Combe C, McCullough KP, Asano Y et al (2004) Kidney Disease Outcomes Quality Initiative (K/DOQI) and the Dialysis Outcomes and Practice Patterns Study (DOPPS): nutrition guidelines, indicators and practices. Am J Kidney Dis 44(Suppl 2):S39-S46

22. Segall L, Mardare N-G, Ungureanu S et al (2009) Nutritional status evaluation and survival in haemodialysis patients in one centre from Romania. Nephrol Dial Transplant 24:2536-2540

23. Małgorzewicz S, Dębska-Ślizień A, Rutkowski B, ŁysiakSzydłowska W (2008) Serum concentration of amino acids versus nutritional status in hemodialysis patients. J Ren Nutr 18:239-247

24. Jatoi A, Yamashita J, Sloan JA et al (2002) Does megestrol acetate down-regulate interleukin-6 in patients with cancer-associated anorexia and weight loss? A North Central Cancer Treatment Group Investigation. Support Care Cancer 10:71-75

25. Schindler AE, Campagnoli C, Druckmann R et al (2003) Classification and pharmacology of progestins. Maturitas 46:s7-s16

26. McCarthy HD, Crowder RE, Dryden S et al (1994) Megestrol acetate stimulates food and water intake in the rat: effects on regional hypothalamic neuropeptide Y concentrations. Eur J Pharmacol 265:99-102

27. Costa A-MN, Spence KT, Plata-Salaman CR et al (1995) Residual $\mathrm{Ca}^{2+}$ channel current modulation by megestrol acetate via a G-protein $\alpha$ s-subunit in rat hypothalamic neurons. J Physiol 487:291-303

28. Barr SI, Janelle KC, Prior JC (1995) Energy intakes are higher during the luteal phase of ovulatory menstrual cycles. Am J Clin Nutr 61:39-43

29. Wallace AM, Kelly A, Sattar N, McArdle CS, McMillan DC (2002) Circulating concentrations of "free" leptin in relation to fat mass and appetite in gastrointestinal cancer patients. Nutr Cancer 44:156-160

30. LaClair R, O'Neal K, Ofner S et al (2008) Precision of biomarkers to define chronic inflammation in CKD. Am J Nephrol 28:808-812

31. Kaysen GA, Dubin JA, Müller HG et al (2000) The acutephase response varies with time and predicts serum albumin levels in hemodialysis patients. Kidney Int 58:346-352

32. Więcek A, Kokot F, Chudek J, Adamczak M (2002) The adipose tissue-a novel endocrine organ of interest to the nephrologist. Nephrol Dial Transplant 17:191-195

33. Stompór T, Sułowicz W, Dembińska-Kieć A et al (2003) An association between body mass index and markers of inflammation: Is obesity the proinflammatory state in patients on peritoneal dialysis? Perit Dial Int 23:79-83

34. Axelsson J, Rashid Qureshi A, Suliman ME et al (2004) Truncal fat mass as a contributor to inflammation in endstage renal disease. Am J Clin Nutr 80:1222-1229

35. Ramos LF, Shintani A, Ikizler TA, Himmelfarb J (2008) Oxidative stress and inflammation are associated with adiposity in moderate to severe CKD. J Am Soc Nephrol 19:593-599

36. Honda H, Rashid Qureshi A, Axelsson J et al (2007) Obese sarcopenia in patients with end-stage renal disease is associated with inflammation and increased mortality. Am J Clin Nutr 86:633-638

37. Beddhu S, Cheung AK, Larive B et al (2007) Inflammation and inverse association of body mass index and serum creatinine with mortality in hemodialysis patients. J Ren Nutr 17:372-380

38. Szeto CC, Kong J, Wu AKL et al (2000) The role of lean body mass as a nutritional index in Chinese peritoneal dialysis patients-comparison of creatinine kinetics method and anthropometric method. Perit Dial Int 20:708-714

39. Dong J, Li Y-J, Lu X-H et al (2007) Correlations of lean body mass with nutritional indicators and mortality in patients on peritoneal dialysis. Kidney Int 73:334-340

40. Mann M, Koller E, Murgo A et al (1997) Glucocorticoidlike activity of megestrol. A summary of Food and Drug Administration experience and a review of the literature. Arch Intern Med 157:1651-1656

41. Andus T, Geiger T, Hirano T et al (1988) Action of recombinant human interleukin 6 , interleukin 1 beta and tumor necrosis factor alpha on the mRNA induction of acute-phase proteins. Eur J Immunol 18:739-745

42. De Feo P, Horber FF, Haymond MW (1992) Meal stimulation of albumin synthesis: a significant contributor to whole body protein synthesis in humans. Am J Physiol 263:E794-E799

43. Thalacker-Mercer AE, Johnson CA, Yarasheski KE et al (2007) Nutrient ingestion, protein intake, and sex, but not age, affect albumin synthesis rate in humans. J Nutr 137: 1734-1740

44. Carrero JJ, Chmielewski M, Axelsson J et al (2008) Muscle atrophy, inflammation and clinical outcome in incident and prevalent dialysis patients. Clin Nutr 27:557-564

45. Kakiya R, Shoji T, Tsujimoto Y et al (2006) Body fat mass and lean mass as predictors of survival in hemodialysis patients. Kidney Int 70:549-556

46. Kato A, Odamaki M, Yamamoto T et al (2003) Influence of body composition on 5 year mortality in patients on regular haemodialysis. Nephrol Dial Transplant 18:333-340

47. Mafra D, Guebre-Egziabher F, Fouque D (2008) Body mass index, muscle and fat in chronic kidney disease: questions about survival. Nephrol Dial Transplant 23:2461-2466

48. Desmeules S, Lévesque R, Jaussent I et al (2004) Creatinine index and lean body mass are excellent predictors of long-term survival in haemodiafiltration patients. Nephrol Dial Transplant 19:1182-1189

49. Ramkumar N, Pappas LM, Beddhu S (2005) Effect of body size and body composition on survival in peritoneal dialysis patients. Perit Dial Int 25:461-469

50. Mulligan K, Zackin R, Von Roenn JH et al (2007) Testosterone supplementation of megestrol therapy does not enhance lean tissue accrual in men with human immunodeficiency virus-associated weight loss: a randomized, double-blind, placebo-controlled, multicenter trial. J Clin Endocrinol Metab 92:563-570

51. Biolo G, Agostini F, Simunic F et al (2008) Positive energy balance is associated with accelerated muscle atrophy and 
increased erythrocyte glutathione turnover during 5 week of bed rest. Am J Clin Nutr 88:950-958

52. Lopes AA, Bragg-Gresham J, Goodkin D et al (2007) Factors associated with health-related quality of life in among hemodialysis patients in the DOPPS. Qual Life Res 16:545-557

53. Mingardi G, Cornalba L, Cortinovis E et al (1999) Healthrelated quality of life in dialysis patients. A report from an Italian study using the SF-36 Health Survey. DIA-QOL Group. Nephrol Dial Transplant 14:1503-1511

54. Spiegel BMR, Melmed G, Robbins S, Esrailian E (2008) Biomarkers and health-related quality of life in end-stage renal disease: a systematic review. Clin J Am Soc Nephrol 3:1759-1768

55. Goller JL, McMahon JM, Rutledge C et al (1997) Dialysis adequacy and self-reported health status in a group of CAPD patients. Adv Perit Dial 13:128-133
56. Kalantar-Zadeh K, Kuwae N, Wu DJ et al (2006) Associations of body fat and its changes over time with quality of life and prospective mortality in hemodialysis patients. Am J Clin Nutr 83:202-210

57. SimicOgrizovic S, Jovanovic D, Dopsaj V et al (2009) Could depression be a new branch of MIA syndrome? Clin Nephrol 71:164-172

58. Kalantar-Zadeh K, Regidor DL, Kovesdy CP et al (2009) Fluid retention is associated with cardiovascular mortality in patients undergoing long-term hemodialysis. Circulation 119:671-679

59. Evans WJ, Reynolds DW (2007) Editorial: megestrol acetate use for weight gain should be carefully considered. J Clin Endocrinol Metab 92:420-421 